\title{
In Vivo Evaluation of Magnetic Targeting in Mice Colon Tumors with Ultra-Magnetic Liposomes Monitored by MRI
}

Caroline J. Thébault ${ }^{1,2,3,4,5}$, Grégory Ramniceanu ${ }^{1,2,3,4}$, Aude Michel $^{5}$, Claire Beauvineau ${ }^{1,2,3,4}$, Christian Girard ${ }^{1,2,3,4}$, Johanne Seguin ${ }^{1,2,3,4}$, Nathalie Mignet ${ }^{1,2,3,4}$, Christine Ménager ${ }^{*}$, BichThuy Doan ${ }^{1,2,3,4^{*}}$

In vivo Magnetic Targeting of Ultra-Magnetic Liposomes

Article

1- Chimie ParisTech, PSL Research University, Unité de Technologies Chimiques et Biologiques pour la Santé (UTCBS), F-75005 Paris, France

2- CNRS, UMR 8258, UTCBS, F-75006 Paris, France

3- Université Paris Descartes, Sorbonne-Paris-Cité, UTCBS, F-75006 Paris, France

4- INSERM, U 1022, UTCBS, F-75006 Paris, France

5- Sorbonne Universités, UPMC Univ Paris 06, CNRS UMR 8234, Laboratoire PHENIX, Case 51, 4 place Jussieu, F-75005 Paris, France

*Corresponding authors, bich-thuy.doan@chimie-paristech.fr ; christine.menager@upmc.fr 


\section{Abstract:}

Purpose: The development of theranostic nanocarriers as an innovative therapy against cancer has been improved by targeting properties in order to optimize the drug delivery to safely achieve its desired therapeutic effect. The aim of this paper is to evaluate the Magnetic Targeting (MT) efficiency of Ultra-Magnetic Liposomes (UML) into CT26 murine colon tumor by MRI.

Procedures: Dynamic Susceptibility Contrast MRI was applied to assess the bloodstream circulation time. A novel semi-quantitative method called $\% \mathrm{I}_{0.25}$, based on the intensity distribution in $\mathrm{T}_{2}{ }^{*}$ weighted MRI images was developed to compare the accumulation of $\mathrm{T}_{2}$ contrast agent in tumors with or without MT. To evaluate the efficiency of magnetic targeting, the percentage of pixels under the intensity value $\mathrm{I}_{0.25}\left(\mathrm{I}_{0.25}=0.25\left(\mathrm{I}_{\max }-\mathrm{I}_{\min }\right)\right)$ was calculated on the intensity distribution histogram.

Results: This innovative method of processing MRI images showed the MT efficiency by a $\% \mathrm{I}_{0.25}$ that was significantly higher in tumors using MT compared to passive accumulation, from $15.3 \%$ to $28.6 \%$. This methodology was validated by ex vivo methods with an iron concentration that is threefold higher in tumors using MT.

Conclusions: We have developed a method that allows a semi-quantitative evaluation of targeting efficiency in tumors, which could be applied to different $\mathrm{T}_{2}$ contrast agents.

Key words: MRI, magnetic targeting, magnetic nanoparticle, liposome, image analysis method, tumor. 


\section{INTRODUCTION}

The last decades have witnessed significant advances on cancer treatment. Chemotherapy still remains the major cancer treatment despite its high toxicity. Recent growth of nanomedicine has enable the development of new nanocarriers for the delivery of drug to their targeting sites and/or triggered features in order to release its properties [1]. The goals of nanocarriers are to improve the benefit to its risk ratio, by increasing the drug accumulation in the tumor, decreasing its accumulation in healthy organs and by the delivery of the therapeutic molecule preferentially to their targeted area [2]. Nanocarriers can be liposomes, nanoparticles, polymers, micelles or antibodies, just to name a few [3]. Among the various nanocarriers, liposomes represent a versatile drug carrier matching with high biocompatibility and their ability to enclose polar drugs in its inner aqueous cavity and hydrophobic or non-polar therapeutic payloads in the lipid bilayer [4]. Due to their functional versatility, liposomes thus remain the most intensively investigated delivery vector. A first strategy to selectively target tumor cells is to graft ligands or antibodies to the liposomal surface. Physical approach is an alternative and complementary approach, which consists in non-invasive magnetic targeting (MT) in order to guide and accumulate magnetic nanomaterials in selected tissues or cells by applying an external magnetic field gradient. Liposomes have been investigated for encapsulating magnetic nanoparticles and several drugs as for instance, doxorubicin, anti-infectious, antiestrogen, and myorelaxants [5].

The strategies that would allow magnetic nanoparticles encapsulation within liposomes has been developed mainly to magnetic resonance imaging (MRI) tracking [4], [6] and magnetic targeting [7]-[9]. For this last purpose, the main issue is to achieve a high degree of loading in order to obtain an important magnetization of the liposomes. We have recently proposed an alternative method to standard thin film hydration process that consists in evaporation of a reverse emulsion (water in oil) that enables a higher internal aqueous loading of magnetic 
nanoparticles (almost a 100-fold). Due to their high magnetic payload, ultra-magnetic liposomes (UML) were able to accumulate in solid tumors via the application of an external magnet [8], [10].

The control of the nanocarrier biodistribution is essential for the development of nanomedicine. The advantage of UML is dual as they can be followed by MRI and guided by using a magnet. MRI is the modality of choice as it is non-invasive and provides quantitative $3 \mathrm{D}$ data with high contrast. Spatial and temporal pharmacokinetics can be easily performed in order to monitor the in vivo biodistribution of diagnostic or therapeutic vectors in organs. This pharmacokinetic is especially important to check the vector stealthiness property that is required to increase tumor accumulation [11]. Several MRI methods based on their relaxation rates $\mathrm{R}_{2}$ and $\mathrm{R}_{2} *$ measurements are developed to quantify the iron content in tissues such as the liver, but the quantification remains a challenge because these relaxometry-based techniques are sensitive to the physiopathological status of the tissue, to the iron particle size and to the magnetic field $\mathrm{B}_{0}$ heterogeneity [12].

The aim of this paper is to propose a new method to evaluate the magnetic targeting efficiency of UML in xenografted murine colon tumors. For this purpose, an original in vivo MRI method of image analysis was developed. The results were compared to ex vivo analysis: iron titration and confocal microscopy observation.

\section{MATERIALS AND EXPERIMENTAL}

\section{Materials}

1,2-dipalmitoyl-sn-glycero-3-phosphocholine ～(DPPC), 1,2-distearoyl-sn-glycero-3phosphocholine (DSPC) and 1,2-distearoyl-sn-glycero-3-phosphoethanolamine-N- 
[amino(polyethylene glycol)-2000] (ammonium salt) (DSPE-PEG2000) were purchased from Avanti Polar lipids, Inc. Cy5.5-NHS ester was purchased from Interchim (Montluçon, France). The CT26 murine colon carcinoma cell line (ATCC, CRL-2638), the BNL 1ME A.7R.1 (ATCC, TIB-75) murine normal epithelial liver cell line and the NIH/3T3 murine fibroblasts cell line (ATCC, CRL-1658) were purchased from American Type Culture Collection (LGC Standards, Molsheim, France). Extravidin-peroxydase was purchased from Sigma-Aldrich. Alamar Blue was supplied from Thermo Fisher Scientific.

\section{MNP synthesis and UML preparation}

Magnetic NanoParticles (MNP) of maghemite $\left(\gamma-\mathrm{Fe}_{2} \mathrm{O}_{3}\right)$ were synthetized according to Massart's procedure [13] (detailed in Supplementary Material) and redispersed at $1 \mathrm{M}$ of iron in injection buffer $(0.11 \mathrm{M} \mathrm{NaCl}, 0.02 \mathrm{M}$ sodium citrate and $0.01 \mathrm{M}$ HEPES) for in vivo experiments.

UML was prepared by reverse phase evaporation method as described by Béalle et al. [8] and detailed in the Supplementary Material.

To synthetize fluorescent UML, Cyanine 5.5-NHS esther was grafted to DSPE-PEG $2000-\mathrm{NH}_{2}$ according to Jarzyna et al. [14] (Supplementary Material).

Iron was quantified by atomic absorption spectroscopy after degradation of UML in $37 \% \mathrm{HCl}$.

\section{Physico-chemical characterizations}

MNP and UML were observed by Transmission Electron Microscopy (TEM) and UML by CryoTEM. UML diameters were measured by Tunable Resistive Pulse Sensing (TRPS) on a qNano system (Izon Science, Oxford, UK).

To evaluate the volume fraction of MNP per UML, the magnetophoretic mobilities of liposomes were measured in a magnetic field gradient of $195 \mathrm{~T} / \mathrm{m}$ [15]. Three independent experiments, each tracking 50 UML, were performed to evaluate the average UML velocity. The balance 
between the magnetic force and the viscosity one were applied on liposomes to allow the calculation of the magnetic load.

\section{MRI system}

Relaxivity measurements were performed on a $7 \mathrm{~T}$ vertical spectrometer fitted with an ultrashielded refrigerated magnet (300WB, Bruker, Avance II, Wissembourg, France), and equipped with an RF birdcage coil with a $40 \mathrm{~mm}$ inner diameter (Bruker) and a nominative 200 mT.m-1 actively shielded gradient coil. The shimming protocol was performed using an optimized combined shimming directions available from our 10 shims micro imaging system, adapted to our micro MRI spectrometer and the $40 \mathrm{~mm}$ diameter Bruker RF probe.

For in vivo MRI experiments, animals were anesthetized with $1.5 \%$ isoflurane gas in an air/ $\mathrm{O}_{2}$ mixture $(0.5 \mathrm{~L} / \mathrm{min}$ and $0.2 \mathrm{~L} / \mathrm{min}$ respectively).

\section{Relaxivity measurement}

Samples of MNP and UML were diluted in injection buffer $(0.108 \mathrm{M} \mathrm{NaCl}, 0.02 \mathrm{M}$ sodium citrate and 0.01 M HEPES) at various concentrations of iron $(0.01,0.05,0.10,0.20,0.50 \mathrm{mM})$. In vitro relaxivity experiments were carried out by recording $\mathrm{T}_{2}$ and $\mathrm{T}_{1}$ maps. The Paravision 5 software allowed the recordings with the following parameters: for $\mathrm{T}_{1}$ map: RARE images ; TE $=13 \mathrm{~ms} ; \mathrm{TR}=15 \mathrm{~s}, 8 \mathrm{~s}, 3 \mathrm{~s}, 1.2 \mathrm{~s}, 0.800 \mathrm{~s}, 0.594 \mathrm{~s}, 0.300 \mathrm{~s}, 0.144 \mathrm{~s}, 0.050 \mathrm{~s}, 0.033 \mathrm{~s}$, RARE factor 2; for $\mathrm{T}_{2}$ map: multi-echo MSME images: Hermitian pulse, TR/TE = $15 \mathrm{~s} / 11 \mathrm{~ms}, 32$ echoes. Fields of view of $3 \times 3 \mathrm{~cm}^{2}$, matrix size of $128 \times 64$ and slice thickness of $1.5 \mathrm{~mm}$ were used for $T_{1}$ and $T_{2}$ maps. Relaxation times $T_{1}$ and $T_{2}$ of each sample were calculated by fitting the $T_{1}$-weighed and $T_{2}$-weighed signal intensity $S$ with the relation $S_{T R}=A+B\left(1-\exp \left(-T R / T_{1}\right)\right.$ and $\mathrm{S}_{\mathrm{TE}}=\mathrm{A}+\mathrm{B} \cdot \exp \left(-\mathrm{TE} / \mathrm{T}_{2}\right)$. Molar relaxivities $\mathrm{r}_{1}$ and $\mathrm{r}_{2}$ were obtained using the following equation $\frac{1}{T_{i}}=r_{i}[\mathrm{Fe}]+\frac{1}{T_{i, 0}}$ with i: 1 or 2 . 


\section{Cytotoxicity assay}

The cytotoxicity was determined on CT26 tumor cells, TIB-75 hepatocytes and NIH/3T3 fibroblasts using the Alamar Blue test as described in the Supplementary Material.

\section{Animals}

Studies were carried out on 7 week old Balb/C female mice (Janvier, St Genest de Lisle, France). Animal experiments were conducted according to European and National guidelines and were approved by the institutional ethics committee (CEEA34.JS.142.1). In vivo experiments presented in this work required 62 animals.

\section{In vivo liver uptake by MRI}

To visualize the capture of UML by the liver, a Dynamic Susceptibility Contrast (DSC) imaging methodology was used: IntraGateFLASH (Fast Low Angle Shot gradient echo sequence with IntraGate) images were recorded to suppress breath motion artifacts. IntraGate module patented by Bruker is a self- gating sequence based on a retrospective image reconstruction by sorting out the MRI signal between the breath peaked motions. [16], [17]. It was used in a cine mode with 1 min duration and adapted time resolution accordingly to the uptake and clearance behavior of the ULMs: Hermitian pulse, TR/TE $=90 \mathrm{~ms} / 3 \mathrm{~ms}, 52$ repetitions. Field of view of $3 \times 3 \mathrm{~cm}^{2}$, matrix size of $256 \times 256$, for a $117 \mu \mathrm{m}$ in plane resolution, and 5 slices distant of 3 $\mathrm{mm}$ with a thickness of $1.088 \mathrm{~mm}$ were used. After 3 reference acquisitions, $100 \mu \mathrm{L}$ of MNP or UML at $5 \mathrm{mM}$ iron concentration in injection buffer were injected using a $30 \mathrm{G}$ catheter previously placed in the caudal vein of the animal. The mouse was monitored by MRI for $1 \mathrm{~h}$ after injection with $1 \mathrm{~min}$ time resolution and then points were taken at $2 \mathrm{~h}, 4 \mathrm{~h}$ and at different times for a week to detect the clearance. For each time, a Region of Interest (ROI) was drawn for the liver and for the water phantom tube located near the mouse into the field of view. The signal of these two ROI was determined using the ImageJ software (National Institutes of Health, Bethesda). The signal of the liver was then normalized with the phantom signal and 
reported for each time point after normalization by the precontrast value. The GraphPad 6 software allowed the fitting of the signal versus time curves using nonlinear regression (bell shaped fitting).

\section{Ectopic tumor implantation}

Two weeks after implantation, a mouse bearing a subcutaneous CT26 tumor was sacrificed. The tumor was removed, submerged in DMEM culture medium and cut in $30 \mathrm{~mm}^{3}$ fragments. For the implantation, the mouse lateral flank above the posterior paws was disinfected using alcohol, the tumor fragments were transferred into sterile PBS and inserted subcutaneously using a 12 gauge trocar into the two mouse flanks sides above the posterior paws [18].

\section{In vivo magnetic targeting studied by MRI}

Whole tumors as reference were imaged with the following sequence: FLASH images: Hermitian pulse, TR/TE $=350 \mathrm{~ms} / 5 \mathrm{~ms}, \alpha=40^{\circ}$, triggered on respiration. Field of view of $3 \mathrm{x}$ $3 \mathrm{~cm}^{2}$, matrix size of $256 \times 256$ corresponding to $177 \mu \mathrm{m} \times 117 \mu \mathrm{m}$ in plane resolution and 15 to 17 slices with a thickness of $1 \mathrm{~mm}$ were used, for an acquisition time of about $7 \mathrm{~min}$. The animal was removed from the MRI apparatus and a catheter (30 G) was placed in its caudal vein. Magnetic targeting was achieved through two cylindrical magnets (NdFeB, with diameter $6 \mathrm{~mm} \times 2 \mathrm{~mm}$ thickness, $0.35 \mathrm{~T}$, Supermagnete, Germany) that was stuck together and positioned on the skin over the tumor; the other contralateral tumor was taken as the control without magnetic targeting. UML (100 $\mu \mathrm{L}$ at $100 \mathrm{mM}$ in iron) were injected through the catheter and the animal was kept in this position for $30 \mathrm{~min}$. Then the magnets were removed and posttargeting MRI images of tumors were acquired. The animals were then sacrificed.

\section{Data processing}

For the data processing, MRI image of each slice was opened in the ImageJ software (National Institutes of Health, Bethesda) using the plugin BrukerOpener, the ROI corresponding to the 
tumor was drawn and the pixel intensity distribution was obtained. The pixel intensity distributions for each slice of the tumor were compiled using MATLAB software (R2015a, Natick, Massachusetts, United States) to obtain a pixel intensity distribution for the whole tumor. The percentage of pixels under the $\mathrm{I}_{0.25}$ value was then calculated $\left(\mathrm{I}_{0.25}=\right.$ $0.25^{*}\left(\right.$ Intensitymax $_{\max }-$ Intensitymin $_{\text {) }}$ ) with the same software (Script in the Supplementary Material). To compare the reference tumor and tumors with or without magnetic guidance, percentages of pixels under the $\mathrm{I}_{0.25}$ for each condition were averaged and a statistic MannWhitney test was performed using GraphPad 6.

\section{ICP analysis}

After harvest, iron was quantified from tumors, liver, spleen, kidneys and lungs by ICP-AES (Inductively Coupled Plasma - Atomic Emission Spectrometry) as described in Supplementary Material.

\section{Biphotonic and Confocal Microscopy}

The fluorescence intensity of tumors after injection of fluorescent UML with or without magnetic targeting was measured ex vivo by using biphotonic and confocal microscopies as described in the Supplementary Material.

\section{RESULTS}

\section{Characterization of $U M L$}

Magnetic NanoParticles (MNP) of maghemite $\left(\gamma-\mathrm{Fe}_{2} \mathrm{O}_{3}\right)$ were sorted by size and the fraction with the higher mean diameter $\left(\mathrm{d}_{0}=9.8 \mathrm{~nm} ; \sigma=0.37\right)$ was chosen to acquire a greater magnetization (Supplementary Material). The particles were stabilized by complexation with citrate molecules and dispersed in an aqueous buffer $(0.108 \mathrm{M} \mathrm{NaCl}, 0.02 \mathrm{M}$ sodium citrate and 0.01 M HEPES) at $\mathrm{pH} 7$ for all the in vivo experiments. Ultra-Magnetic Liposomes (UML) and fluorescent UML were prepared by reversed phase evaporation process (REV) [8]. After 
magnetic separation to eliminate non-encapsulated MNP, the iron concentration of UML was measured by flame ICP spectrometry. TEM observation confirmed that the UML were filled with MNP (Figure1-a). The presence of the bilayer has been confirmed by cryoTEM proving the formation of unilamellar liposomes (Supplementary Material). Dynamic light scattering (DLS) was not appropriate to characterize UML encapsulating MNP since the mean diameter reflects both MNP and UML sizes. Moreover, since the refractive index of both nano-objects were different, the values were not relevant. Therefore, Tunable Resistive Pulse Sensing (TRPS) method [19] was used for the measurement of UML diameters. As the liposome size was obtained by counting each individual liposome that went through a calibrated pore, both diameter and number of UML could be obtained (Figure1-b). The availability of membranes with the TRPS method that starts with nanopores at $50 \mathrm{~nm}$, does not allow this technique to measure the MNP. According to a log-normal law, the mean diameter was determined at 230 nm with a 0.2 polydispersity.

\section{Figure 1}

In order to determine the amount of MNP per UML, magnetophoresis experiments were carried out. The velocity of UML submitted to a magnetic field gradient was measured. Therefore, volume fraction of MNP per UML can be calculated to counterbalance the forces applied between viscosity and magnetic forces applied on UML [15]. With a mean velocity of 37.6 $\mu \mathrm{m} / \mathrm{s}$ in a $195 \mathrm{~T} / \mathrm{m}$ magnetic field gradient, the volume fraction of MNP in UML was $24 \%$ which indicates that about 3,100 MNP were encapsulated per UML (6.1.10-15 $\mathrm{g}$ of Fe/ UML). The molecular ratio of Fe/lipid was calculated as $83(\mathrm{~mol} / \mathrm{mol})$, which is higher than data reported in literature $(0.53 \mathrm{~mol} \mathrm{Fe} / \mathrm{mol}$ lipid [20], $19 \mathrm{~mol} \mathrm{Fe} / \mathrm{mol}$ lipid [21], $0.15 \mathrm{~mol} \mathrm{Fe} / \mathrm{mol}$ lipid [22]). This high MNP loading is crucial to achieve magnetic targeting. Indeed, the magnetic force $\mathrm{F}_{\text {mag }}$ depends on the UML magnetic moment and thus on the number of MNP entrapped in the liposome. 


\section{$\underline{\text { Table } 1}$}

Relaxivities in solution were measured at 7 Tesla for MNP and UML and they were compared to Cliavist ${ }^{\circledR}$, a commercial iron oxide MRI contrast agent. The values of longitudinal and transverse relaxivities $r_{1}$ and $r_{2}$ are given for these three types of objects in Table 1. Iron oxide nanoparticles are well known for their contrast agent properties in $\mathrm{T}_{2}$ weighted MR Imaging. They give a hyposignal in appropriate $T_{2}$ weighted MRI acquisition sequences. The UML transverse relaxivity $r_{2}$ increases compared to the one of free MNP. Indeed, once there is compartmentalization in liposomes, cooperating MNP give a higher magnetic moment resulting in a higher proton spin dephasing with shortened $\mathrm{T}_{2}$ value [23]. UML relaxivities are therefore suitable for in vivo contrast agent use with a better $\mathrm{r}_{2} / \mathrm{r}_{1}$ of 150 compared to 78 for the commercial Cliavist ${ }^{\circledR}$ and 35 for free MNP. Taking into consideration the difference of $\mathrm{r}_{2}$ values between free MNP and UML, we used relaxivity measurements to evaluate the stability of UML over time. A stable $\mathrm{r}_{2}$ value reflecting the UML stability in RPMI was observed for at least $5 \mathrm{~h}$ (Supplementary Material).

In order to confirm that these UML were appropriate for intravenous injections, cytotoxicity tests were performed in this RPMI medium. Viabilities of CT26 (colon tumor), TIB-75 (hepatocyte) and NIH/3T3 (fibroblast) cells were evaluated using increasing concentrations of UML. The absence of UML toxicity at iron concentration under $10 \mathrm{mM}$ for tumor and fibroblast cells, and a low toxicity for hepatocyte cells, were observed as illustrated in Figure 2. From the stability and toxicity results, we considered that in vivo injections were conceivable.

\section{$\underline{\text { Figure } 2}$}

\section{In vivo liver uptake}

In vivo biodistribution studies were then performed to characterize the kinetic behavior of the UML in mice and, in particular, to assess the stealthiness property of UML after intravenous 
injection. To ascertain tumor magnetic targeting, the UML must indeed circulate within a certain time into the bloodstream before being eliminated by Kupffer cells and captured by the liver and spleen as expected from their size. The Dynamic Susceptibility Contrast MR imaging at $7 \mathrm{~T}$ allowed the following of the hepatic uptake by kinetic acquisitions. These acquisitions can be specifically adjusted every minute for the first hour and sequentially for a week to obtain the whole process of nanoparticle elimination: liver uptake, and degradation [11]. Figure 3 summarizes the kinetic of capture and clearance of MNP and UML with examples of MRI images illustrating the evolution of signal versus time at each specific point (1: initial signal, 2 : decreasing slope, 3: plateau, 4: increasing signal). The UML and MNP uptake by the liver resulted in a decrease of MRI signal in the liver up to a minimum. To obtain a match between this minimum of signal and the maximum of liver uptake, $5 \mathrm{mM}$ of iron was injected as previously determined by Ramniceanu et al. [11]. Indeed, with a higher concentration of iron, the MRI signal in the liver saturated, preventing the visualization of the minimum value corresponding to the maximum uptake by the liver. After a plateau of a few days, the signal increased corresponding to the liver clearance.

\section{Figure 3}

From hundred nanometer size of nanocarriers, a very fast hepatic capture was expected. In our case, the PEG corona around UML membrane allowed an improvement of the circulation time [1]. Indeed, the minimum of signal corresponding to the total uptake of UML by the liver was measured at $1 \mathrm{~h}$ after injection. Furthermore, after $10 \mathrm{~min}$, the signal intensity was at $50 \%$ between its minimum and maximum, meaning that a significant part of the UML was still circulating, although there is no linear correlation but an exponential correlation between the MRI intensity and the MNP concentration Therefore, the circulation duration in the bloodstream and within the tumor vascular network was about 30 min during which the 
magnetic targeting can be expected to be efficient. The difference of minimum $\mathrm{T}_{2}{ }^{*}$ weighted MRI signal value between MNP and UML is due to the difference of transverse relaxivity $\mathbf{r}_{2}$.

\section{In vivo magnetic targeting}

MT efficiency was investigated on mice with bilateral CT26 murine tumors implanted on each flank. After a $\mathrm{T}_{2}{ }^{*}$-weighted MRI image acquisition pre-injection, two superimposed magnets were positioned on one of the tumors and UML were injected intravenously. The magnetic field at the surface was $0.35 \mathrm{~T}$ and then decreased exponentially according to the following equation: $B=0.354 e^{-0.41 d}-0.004$ with $\mathrm{B}$ the magnetic field in Tesla and $\mathrm{d}$ the distance from magnets in $\mathrm{mm}$. To compare the passive accumulation of UML in tumors due to Enhanced Permeability Retention (EPR) effect and the effect of MT, magnets were removed after 30 min and a $\mathrm{T}_{2}{ }^{*}-$ weighted MRI image was acquired. Figure 4 shows an example of pre and post-injection images. The difference of accumulation was observed with a higher hyposignal in the tumor using MT.

\section{Figure 4}

To evaluate the in vivo increase of UML accumulation in tumors, from MT, a post processing methodology called \% $\mathrm{I}_{0.25}$ was developed. Regions Of Interest (ROI) were drawn on each slice of the tumor on MRI images (figure 5-a and c) and the pixel intensity distribution was obtained for each slice. The compilation of these distributions gave a single pixel intensity distribution per tumor as shown in figure 5-b and d.

\section{Figure 5}

The mean signal in $\mathrm{T}_{2}{ }^{*}$-weighted images did not show significant differences between tumors injected with UML with MT (9 375 u.a. \pm 1 017) or without MT (10 335 u.a. \pm 1 868). Then, 
the percentage of pixels under the $\mathrm{I}_{0.25}\left(0.25\right.$ (Intensitymax-Intensity $\left.\mathrm{min}_{\min }\right)$ ) for tumors before and after injection, with or without MT was compared (figure 5-e). This parameter was chosen as the UML presence as $\mathrm{T}_{2}$ contrast agent induced a shift in the intensity distribution toward lowintensity values. A significant difference was obtained between non-injected mice and injected mice, $2.9 \%$ and $15.3 \%$ respectively. This reflected the passive accumulation of UML in these highly vascularized tumors [24]. More interestingly, the active accumulation correlated to magnetic targeting showed a significant increase as referred to UML injected mice, meaning a net gain when compared to passive accumulation. $28.6 \%$ of the pixels were under the $\mathrm{I}_{0.25}$ in the case of MT active accumulation compared to $15.3 \%(*)$ for the passive accumulation.

This methodology of data treatment from signal distribution allowed the analysis of nonhomogenous MRI signal on which global intensity average is not significant enough for the in vivo monitoring of magnetic nanoparticles. Therefore, this methodology can be used to compare diverse targeting methods with other $\mathrm{T}_{2}$ nanocarriers and different tumor types.

\section{Ex vivo magnetic targeting}

The accumulation of fluorescent UML (Cyanine 5.5 labeled lipids) in tumors can be checked ex vivo by confocal microscopy and iron titration (Supplementary Material). The signal measured from tumors with MT $\left(12.10^{5}\right)$ was significantly higher $(* *)$ than the signal from tumors with passive accumulation $\left(8.4 .10^{5}\right)$. Furthermore, ex vivo ICP measurements performed on tumors revealed a 3 fold increase of the iron accumulation in tumors with MT $(13.6 \mu \mathrm{g} / \mathrm{g}$ of tumor) as compared to tumors without MT (3.8 $\mu \mathrm{g} / \mathrm{g}$ of tumor). The increase in both the iron and the lipid concentrations in the targeted tumors suggested that the UML were well preserved during their circulation.

Ex vivo experiments confirmed results obtained in vivo with MRI data treatment and in vivo monitoring methodology. Therefore, the comparison of various targeting techniques can be 
accomplished in vivo by using nanoparticles with $\mathrm{T}_{2}$ MRI contrast agent properties and MRI monitoring.

\section{DISCUSSION}

The quantitative evaluation of a targeting efficiency still remains a challenge. To solve this problem, chemists have to develop carriers/nanoparticles that can be detected by various imaging modalities. However, only few imaging modalities provide an absolute quantitative evaluation. Positron Emission Tomography (PET) is probably the most powerful method because radiotracers allow absolute quantification of the uptake of the positron emitting radiopharmaceuticals with high sensitivity as the detection limit is in the picomolar range. Magnetic nanoparticles and magnetic systems have already been conjugated with PET radiotracers [25]. However, the strategies rely on the incorporation of a specific chelator inside or on the surface of nanoparticles involving constraints related to the manipulation of radioactive species. These systems are not competitive with existing clinical protocols [26][28]. Computed tomography is also well known for its quantitative feature for perfusion in vivo using CT contrast agents like Ultravist and iodine. This modality is ionizing and requires a compromise between patient dose and signal-to-noise ratio [29]. In vivo optical imaging lacks the accurate quantitative feature as light scattering and quenching problems occur and this requires image processing reconstruction to correct tissue absorption [30].

This is why the development of new methodologies that enable the evaluation of magnetic targeting efficiency is crucial.

MRI is a non-invasive, non-ionizing, quantitative, highly contrasted and resolved imaging modality. However, although quantitation has already been demonstrated to be efficient with Gadolinium contrast agents, an image processing method has to be developed for iron oxide 
quantification [31]. Other authors have proposed in vivo quantification methods to access the accumulation of MNP in the tumor. One technique was to calculate the signal decay rate and the relative standard deviation (or relative dispersion) from $T_{2}$ maps to evaluate the heterogeneous distribution of MNP in the tissue [32]. An alternative was to consider the percentage change of the initial relaxivity value $\mathrm{dR}_{2}$ [33]. Choudhury et al. proposed to segment hyposignals induced by the accumulation of MNP in different tumors to take into account the signal variability of the tumor type [34]. They proposed a processing method based on local contrast levels with the calculation of a fraction of the local mean signal intensity which was function of the tumor and they suppressed the regions of natural hyposignal for the calculation of iron oxide nanoparticles uptake.

Quantitative Susceptibility Mapping (QSM) is a method [35] provided from 3D multi-echo sequence, to obtain a quantitative map of susceptibility. The susceptibility is proportional to the iron concentration. It has been applied in our present data but the QSM - MEDI reconstruction algorithm was sensitive to the required high 3D isotropic resolution of the quantitative 3D GE image and no significative difference between the QSM maps with and without magnetic targeting was observed (data not shown). The $\% \mathrm{I}_{0.25}$ appeared to be more robust and easy to use to provide evidence on the magnetic targeting of the UML.

In the present study, we have developed an original method that allowed a semi-quantitative evaluation of targeting efficiency in tumors, a method that could be applied for different $\mathrm{T}_{2}$ contrast agents compared to the EPR effect. This method is based on the modification of the intensity distribution in standard $\mathrm{T}_{2}{ }^{*}$ weighted images, easy to set up in all MRI facility and with short acquisition time. The MNP provided a hyposignal inducing a shift of the intensity histogram towards the low intensity region. Therefore, we chose the percentage of pixels under the $\mathrm{I}_{0.25}$ as the relevant parameter to quantify the increase of MNP accumulation. As our 
processing method compared pre- with post-contrast injection images at $1 \mathrm{~h}$ period acquisition, it could be used in different kinds of tumors with low necrotic features.

Magnetic targeting appears as an alternative and attractive method of targeting compared to active targeting (with ligands). Préat et al. showed, for example, a percentage of injected dose in tumor 2-fold higher with MT than with $\alpha_{v} \beta_{3}$ integrin active targeting using RGD [36].

We demonstrated that UML combine two properties on the same object: they are very good candidates for MT according to the high concentration of MNP in their core and they are also very efficient $\mathrm{T}_{2}$ MRI contrast agent useful to monitor their biodistribution. As shown in this work, UML have a circulation time of about one hour allowing their accumulation into the tumors that can be used for future therapeutic purpose.

At this stage, it would be a particular interest to encapsulate and be able to release actively drugs which will be able to diffuse due to its low molecular weight. Thanks to the ability of liposomes to allow the encapsulation of hydrophilic or hydrophobic drugs, UML can be considered for theranostic. Indeed, several authors have shown the liposomal co-encapsulation of doxorubicin with iron oxide nanoparticles [37], Gd chelates [38] or the encapsulation of a hydrophobic sensitizer with iron oxide nanoparticles [39].

\section{CONCLUSION}

A novel semi-quantitative method based on the intensity distribution in MRI $\mathrm{T}_{2}{ }^{*}$ weighted images was developed to compare in vivo the accumulation of $\mathrm{T}_{2}$ contrast agent. This method was perfectly adapted to evaluate the magnetic targeting efficiency of ultra-magnetic liposomes in CT26 colon tumors. Confocal microscopy and ICP were used as ex vivo techniques to validate this intensity distribution approach. Compared to active targeting, MT is versatile 
because it does not depend on the biological target and is also very easy to handle and cheap. Finally, these UML can be easily designed for theranostic applications.

\section{ACKNOWLEDGEMENTS}

This work was supported by the LabEx MiChem part of French state funds managed by the ANR within Le Programme Investissements d'Avenir under reference ANR-11-IDEX-000402. In vivo imaging was performed at the Life Imaging Facility of Paris Descartes University (LIOPA from the Plateform Imageries du Vivant - PIV) and partly supported by CNRS and ENSCP, ANR LightLab program.

We are grateful to Institut Français Weizmann for a postdoctoral grant (GR), Emmanuel Aubry from ALIPP6 for ICP analysis, Claire Wilhelm for magnetophoresis experiments, Cellular Imaging facility Imagic of Institut Cochin for confocal microscopy and to Jean-Michel Guigner for CryoTEM.

\section{CONFLICT OF INTEREST STATEMENT}

The authors declare that there is no conflict of interest regarding the publication of this paper.

\section{REFERENCES}

[1] Shi J, Kantoff PW, Wooster R, Farokhzad OC (2016) Cancer nanomedicine: progress, challenges and opportunities. Nat. Rev. Cancer 17: 20-37

[2] Mura S, Nicolas J, Couvreur P (2013) Stimuli-responsive nanocarriers for drug delivery. Nat. Mater 12: 991-1003

[3] Lammers T, Aime S, Hennink WE, Storm G, Kiessling F (2011) Theranostic Nanomedicine. Acc. Chem. Res 44: 1029-1038 
[4] Soenen SJ, Velde GV, Ketkar-Atre A, Himmelreich U, De Cuyper M (2011) Magnetoliposomes as magnetic resonance imaging contrast agents. Wiley Interdiscip. Rev. Nanomed. Nanobiotechnol. 3: 197-211

[5] Pattni BS, Chupin VV, Torchilin VP (2015) New Developments in Liposomal Drug Delivery. Chem. Rev. 115: 10938-10966

[6] Amstad E, Kohlbrecher J, Müller E, Schweizer T, Textor M, Reimhult E (2011) Triggered Release from Liposomes through Magnetic Actuation of Iron Oxide Nanoparticle Containing Membranes. Nano Lett. 11 : 1664-1670

[7] Mikhaylov G, Mikac U, Magaeva AA et al. (2011) Ferri-liposomes as an MRI-visible drug-delivery system for targeting tumours and their microenvironment. Nat. Nano technol. $6: 594-602$

[8] Béalle G, Di Corato R, Kolosnjaj-Tabi J et al. (2012) Ultra Magnetic Liposomes for MR Imaging, Targeting, and Hyperthermia. Langmuir, 28 : 11834-11842

[9] Marie H, Lemaire L, Franconi F et al. (2015) Superparamagnetic Liposomes for MRI Monitoring and External Magnetic Field-Induced Selective Targeting of Malignant Brain Tumors. Advanced Functional Materials 25: 1258-69.

[10] Fernandez-Sanchez ME, Barbier S, Whitehead J et al. (2015) Mechanical induction of the tumorigenic $\beta$-catenin pathway by tumour growth pressure. Nature $523: 92-95$

[11] Ramniceanu G, Doan BT, Vezignol C et al. (2016) Delayed hepatic uptake of multiphosphonic acid poly(ethylene glycol) coated iron oxide measured by real-time magnetic resonance imaging. RSC Adv. 6: 63788-63800

[12] Hernando D, Levin YS, Sirlin CB, and Reeder SB (2014) Quantification of liver iron with MRI: State of the art and remaining challenges. J. Magn. Reson. Imaging 40: 1003-1021

[13] Massart R (1981) Preparation of aqueous magnetic liquids in alkaline and acidic media. IEEE $17: 1247-1248$ 
[14] Jarzyna PA, Skajaa T, Gianella A et al. (2009) Iron oxide core oil-in-water emulsions as a multifunctional nanoparticle platform for tumor targeting and imaging. Biomaterials 30: 6947-6954

[15] Wilhelm C, Gazeau F, Bacri JC (2002) Magnetophoresis and ferromagnetic resonance of magnetically labeled cells. Eur. Biophys. J. 31: 118-125

[16] Heijman E, de Graaf W, Niessen P et al. (2007) Comparison between prospective and retrospective triggering for mouse cardiac MRI. NMR Biomed. 20:439-47

[17] Bovens SM, te Boekhorst BC, den Ouden K et al. (2011) Evaluation of infarcted murine heart function: comparison of prospectively triggered with self-gated MRI. NMR Biomed. 24: $307-15$

[18] Seguin J, Doan BT, Latorre Ossa H et al. (2013) Evaluation of Nonradiative Clinical Imaging Techniques for the Longitudinal Assessment of Tumour Growth in Murine CT26 Colon Carcinoma. Int. J. Mol. Imaging 2013: 1-13

[19] Weatherall E, Willmott GR (2015) Applications of tunable resistive pulse sensing. Analyst 140: $3318-3334$

[20] Martina MS, Fortin JP, Ménager C et al. (2005) Generation of Superparamagnetic Liposomes Revealed as Highly Efficient MRI Contrast Agents for in Vivo Imaging. J. Am. Chem. Soc. 127: 10676-10685

[21] Bulte JWM, de Cuyper M, Despres D, Frank JA (1999) Preparation, relaxometry, and biokinetics of PEGylated magnetoliposomes as MR contrast agent. J. Magn. Magn. Mater. 194: 204-209

[22] Lorenzato C, Oerlemans C, van Elk M et al. (2016) MRI monitoring of nanocarrier accumulation and release using Gadolinium-SPIO co-labelled thermosensitive liposomes: Gd-TSM for nanocarrier localization and monitoring of release using MRI. Contrast Media Mol. Imaging 11: 184-194 
[23] Larsen BA, Haag MA, Serkova NJ, Shroyer KR, Stoldt CR (2008) Controlled aggregation of superparamagnetic iron oxide nanoparticles for the development of molecular magnetic resonance imaging probes. Nanotechnology 19: 265102

[24] Seguin J, Nicolazzi C, Mignet N, Scherman D, and Chabot G (2012) Vascular density and endothelial cell expression of integrin alpha v beta 3 and E-selectin in murine tumours. Tumor Biol. 33: 1709-1717

[25] Malinge J, Géraudie B, Savel P et al. (2017) Liposomes for PET and MR Imaging and for Dual Targeting (Magnetic Field/Glucose Moiety): Synthesis, Properties, and in Vivo Studies. Mol. Pharm. 14: 406-414

[26] Seo JW, Zhang H, Kukis DL, Meares CF, and Ferrara KW (2008) A Novel Method to Label Preformed Liposomes with (CU)-C-64 for Positron Emission Tomography (PET).Imaging. Bioconjug. Chem. 19: 2577-2584

[27] Petersen AL, Binderup T, Rasmussen P et al. (2011) 64Cu loaded liposomes as positron emission tomography imaging agents. Biomaterials 32: 2334-2341

[28] Phillips WT, Goins BA, and Bao A (2009) Radioactive liposomes. Wiley Interdiscip. Rev.-Nanomedicine Nanobiotechnology 1: 69-83

[29] Klotz E, König M (1999) Perfusion measurements of the brain: using dynamic CT for the quantitative assessment of cerebral ischemia in acute stroke. Eur. J. Radiol. 30: 170-184

[30] Pesnel S, Akkoul S, Ledée R et al. (2011) Use of an Image Restoration Process to Improve Spatial Resolution in Bioluminescence Imaging. Mol. Imaging 10: 446-452

[31] Brown RW, Cheng YCN, Haacke EM, Thompson MR, Venkatesan R (2014) Magnetic Resonance Imaging: Physical Principles and Sequence Design. John Wiley \& Sons

[32] Oakes JM, Breen EC, Scadeng M, Tchantchou GS, Darquenne C (2014) MRI-based measurements of aerosol deposition in the lung of healthy and elastase-treated rats. J. Appl. Physiol. 116: 1561-1568 
[33] Chertok B, Moffat BA, David AE et al. (2008) Iron oxide nanoparticles as a drug delivery vehicle for MRI monitored magnetic targeting of brain tumors. Biomaterials 29: 487-496

[34] Melemenidis S, Jefferson A, Ruparelia N et al. (2015) Molecular Magnetic Resonance Imaging of Angiogenesis In Vivo using Polyvalent Cyclic RGD-Iron Oxide Microparticle Conjugates. Theranostics 5: 515-529

[35] Wang, Y.; Liu, T. (2015) Quantitative susceptibility mapping (QSM): Decoding MRI data for a tissue magnetic biomarker: QSM. Magnetic Resonance in Medicine 73, 82-101

[36] Schleich N, Po C, Jacobs D et al. (2014) Comparison of active, passive and magnetic targeting to tumors of multifunctional paclitaxel/SPIO-loaded nanoparticles for tumor imaging and therapy. J. Controlled Release 194: 82-91

[37] Chen J, Ke X, He Z et al. (2012) A MSLN-targeted multifunctional nanoimmunoliposome for MRI and targeting therapy in pancreatic cancer. Int. J. Nanomedicine 7: 5053-5065

[38] de Smet M, Heijman E, Langereis S, Hijnen NM, Grüll H (2011) Magnetic resonance imaging of high intensity focused ultrasound mediated drug delivery from temperaturesensitive liposomes: An in vivo proof-of-concept study. J. Controlled Release 150: 102110

[39] Di Corato R, Béalle G, Kolosnjaj-Tabi J et al. (2015) Combining Magnetic Hyperthermia and Photodynamic Therapy for Tumor Ablation with Photoresponsive Magnetic Liposomes. ACS Nano 9: 2904-2916

\section{Table}

Table 1: Relaxivities values of MNP, UML and Cliavist ${ }^{\circledR}$ in solution at $7 \mathrm{~T}$.

\begin{tabular}{|c|c|c|c|}
\hline & MNP & UML & Cliavist $^{\circledR}$ \\
\hline $\mathbf{r}_{1}\left(\mathbf{m M}^{\mathbf{1}} \cdot \mathbf{s}^{\mathbf{- 1}}\right)$ & 2.2 & 1.5 & 1.3 \\
\hline $\mathbf{r}_{2}\left(\mathbf{m M}^{-\mathbf{1}} \cdot \mathbf{s}^{\mathbf{- 1}}\right)$ & 77 & 225 & 202 \\
\hline $\mathbf{r}_{2} / \mathbf{r}^{1}$ & 35 & 150 & 78 \\
\hline
\end{tabular}




\section{Figure Legends}

Figure 1: (a) TEM picture of UML; (b) UML size distribution measured by TRPS

Figure 2: Viability of CT26 (colon tumor), TIB-75 (hepatocyte) and NIH/3T3 (fibroblast) as a function of iron concentration after $1 \mathrm{~h}$ incubation with UML.

Figure 3: MRI kinetic visualization of liver uptake, and liver clearance after intravenous injection of MNP or UML. (a) In vivo IntraGateFLASH MRI images of the liver before and at $30 \mathrm{~min}, 120 \mathrm{~min}$ and 15 days after injection of MNP or UML. (b) Kinetic curves of hepatic uptake after intravenous injection of MNP (blue) or UML (red) with 3 animals involved in each group. Lines connecting points are a guide for the eye.

Figure 4: In vivo $\mathrm{T}_{2}{ }^{*}$-weighted MRI images of CT26 tumors (a) before and (b) after UML injection with and without Magnetic Targeting (MT)

Figure 5: $\% \mathrm{I}_{0.25}$ methodology of data processing from the $\mathrm{T}_{2}{ }^{*}$ weighted MRI images for evaluation of in vivo UML accumulation in tumors. (a) Tumor before injection; (b): MRI intensity distribution associated to the tumor before injection; (c) tumor after UML injection; (d): MRI intensity distribution associated to the tumor after UML injection and Magnetic Targeting (MT); (e) Summary of percentage of pixels under $\mathrm{I}_{0.25}$ on MRI tumor images. Non gaussian distribution Mann-Whitney test: *: $\mathrm{P}<0.05 / * *$ : $\mathrm{P}<0.01 / * * *: \mathrm{P}<0.001 / * * * *: \mathrm{P}<0.001 /$ ns: $\mathrm{P}>0.5$ 